\title{
Genera of Vertex Operator Algebras and three dimensional Topological Quantum Field Theories
}

\author{
Gerald Höhn *
}

\begin{abstract}
The notion of the genus of a quadratic form is generalized to vertex operator algebras. We define it as the modular braided tensor category associated to a suitable vertex operator algebra together with the central charge. Statements similar as known for quadratic forms are formulated.

We further explain how extension problems for vertex operator algebras can be described in terms of the associated modular braided tensor category.
\end{abstract}

\section{Introduction}

This note is a write-up of talks given at the workshop "Vertex Operator Algebras in Mathematics and Physics" at the Fields Institute in Toronto in October 2000 and at some other occasions before. Its purpose is to explain how results in low dimensional quantum field theory obtained from different perspectives and with different motivations can be put into a uniform picture by generalizing the notions of discriminant forms and genera from integral quadratic forms to vertex operator algebras, indicating a rich underlying arithmetical theory.

The relation between two-dimensional conformal field theory and three-dimensional quantum field theory has been quite known from the beginning. For example, Witten relates in his well-known paper Wit89 the Wess-Zumino-Witten models with Chern-Simons theories and the Jones polynomial of knots. The basic idea is to use the monodromy properties of correlation functions on surfaces to obtain invariants for knots and three-dimensional manifolds.

Being not overly precise, one can say that a quantum field theory is a functor from some bordism category of oriented manifolds to some category of linear spaces satisfying some basic gluing axioms.

*Mathematisches Institut, Eckerstraße 1, 79104 Freiburg, Germany, gerald@mathematik.uni-freiburg.de 


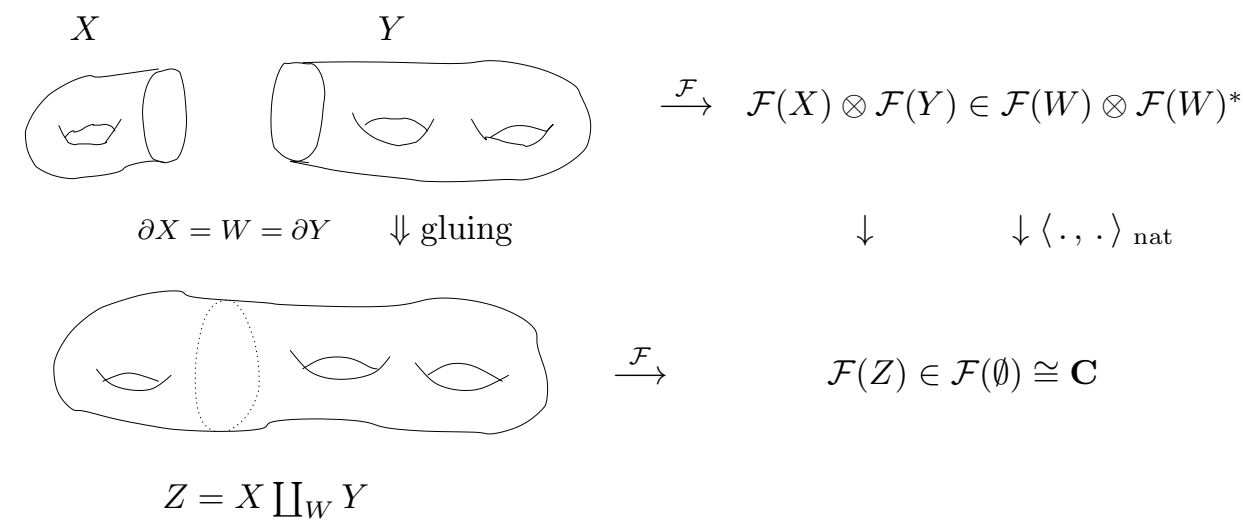

For 2d-CFTs, one takes two-dimensional surfaces with a conformal structure on it and one is forced to take infinite dimensional linear spaces. Using instead Riemannian surfaces (which have a naturally defined conformal structure), one reaches the more restricted but essential notion of chiral algebras. For three-dimensional topological quantum field theories (3dTQFTs), one takes three-dimensional topological manifolds (together with extra structures like framed links) and only finite dimensional linear spaces.

It took some time before precise and efficient mathematical formulations for both structures, originating partly from physics, emerged. Chiral algebras have been axiomatized by the algebraic structure of vertex algebras; see Bor86, FLM88, Seg89, Hua97, Kac97, FBZ2001, BD for some approaches. 3d-TQFTs can be constructed from modular braided tensor categories; see MS89, Ati90, Tur94, Boj2001.

In this paper, we will restrict ourselves mainly to vertex operator algebras (VOAs); these are vertex algebras which have a Virasoro element and we assume that they are nonnegatively graded; we consider only simple and rational VOAs and make some further strong assumptions. There are many different kinds of examples of VOAs known, but it is quite unclear yet how a general structure theory for them may look like.

An important class of VOAs can be constructed from positive definite even integral lattices. For integral lattices, there is a deeply developed structure theory available leading to invariants like rational equivalence, the genus, or the spinor genus. The first purpose of this paper is to explain how the notion of the genus can be generalized in a natural way from lattices to VOAs by using modular braided tensor categories so that the definition is compatible with the notion of the genus for lattices. Secondly, we show that new VOAs can be constructed from given ones only by using the associated modular braided tensor category, generalizing thereby the well-known discriminant technique of quadratic form theory; cf. Höh97].

It should be mentioned that there are papers, which do not distinguish between VOAs and the associated modular braided tensor category. Usually, the authors make Assumption 3.2 and work in the setting of 3d-TQFTs. The example of the lattice VOAs associated to the two positive definite unimodular lattices $E_{8}^{2}$ and $D_{16}^{+}$in dimension 16 Kne57 shows that there are different VOAs which can lead to the same 3d-TQFT.

The paper is organized as follows. In the second section, some basic definitions and results from the theory of integral quadratic forms are recalled. In section three, we define the genus of a vertex operator algebra and try to formulate theorems or at least conjectures analogous to the ones known for quadratic forms. In the final section, we reformulate the 
extension problem for vertex operator algebras in terms of the associated modular braided tensor category as already outlined in my paper Höh97. This extension problem can be considered as a kind of problem in coding theory over 3d-TQFTs. The solution for VOAs defining an abelian intertwining algebra is given. Also, complete results for self-dual framed VOAs or, equivalently, self-dual codes over the 3d-TQFT associated to the Ising model, are presented.

There are many open problems about the structure of vertex algebras compared to what is known for quadratic forms. But the theory of quadratic forms is more than two hundred years old. It seems to me that from the fifteen year old theory of vertex algebras we just can see only the tip of the iceberg of such a structure theory.

I like to thank the organizers of the conference for inviting me to the Fields Institute and for the possibility to present these ideas. I am grateful to Markus Rosellen for his comments on an early version of the paper and for discussions about abelian intertwining algebras as well as to Geoffrey Mason for reminding me of some references. I also like to thank Jim Lepowsky and the referee for their many valuable suggestions. Finally, my thanks goes to Supun Koranasophonpun for proofreading the manuscript.

\section{Genera of integral quadratic forms}

We collect some results from the arithmetic of integral quadratic forms. The theory of genera for binary forms was developed by Gauß in Gau1801. Instead of the more classical notion of quadratic forms in coordinates, we use the language of lattices.

We denote in this section by $\mathbf{Z} / N \mathbf{Z}$ the additive group of the integers modulo $N$ and by $\mathbf{Z}_{p}$ the ring of $p$-adic integers. Recall that a map $q$ from an abelian group $B$ into an additively written group $G$ is called a quadratic form if the expression $q(x+y)-q(x)-q(y)$ is additive in $x$ and in $y$, i.e., bilinear.

Definition 2.A A finite quadratic space $(A, q)$ is a finite abelian group $A$ together with a quadratic form $q: A \longrightarrow \mathbf{Q} / 2 \mathbf{Z}$, such that the induced bilinear form $b: A \times A \longrightarrow \mathbf{Q} / \mathbf{Z}$, $b(x, y)=\frac{1}{2}(q(x+y)-q(x)-q(y))$ is nondegenerate. Note that different quadratic forms can induce the same bilinear form.

Theorem 2.1 (Structure theorem for finite quadratic spaces; cf. [Nik80], Sec. 8) A finite quadratic space can be decomposed uniquely as a direct sum:

$$
(A, q)=\bigoplus_{p \| A \mid}\left(A_{p}, q_{p}\right),
$$

where the $A_{p}$ are the $p$-Sylow subgroups of $A$ and $q_{p}$ is the quadratic form $q$ restricted to $A_{p}$. Furthermore, each $\left(A_{p}, q_{p}\right)$ is the direct sum of quadratic spaces as follows:

For primes $p>2$, it is the sum of spaces $\left(\mathbf{Z} / p^{k} \mathbf{Z}, q_{\theta}\right)$, with $k \geq 1, \theta \in\{ \pm 1\}$ and quadratic form $q_{\theta}: \mathbf{Z} / p^{k} \mathbf{Z}=\langle x\rangle \longrightarrow \mathbf{Q} / 2 \mathbf{Z}, q(x)=2 c_{\theta} / p^{k}$, where $c_{\theta}$ is an integer with Jacobi symbol $\left(\frac{c_{\theta}}{p}\right)=\theta$. The only relation in the free abelian group generated by the $\left(\mathbf{Z} / p^{k} \mathbf{Z}, q_{\theta}\right)$ for fixed $p$ is $\left(\mathbf{Z} / p^{k} \mathbf{Z}, q_{\theta}\right) \oplus\left(\mathbf{Z} / p^{k} \mathbf{Z}, q_{\theta}\right) \cong\left(\mathbf{Z} / p^{k} \mathbf{Z}, q_{\theta^{\prime}}\right) \oplus\left(\mathbf{Z} / p^{k} \mathbf{Z}, q_{\theta^{\prime}}\right)$, with $\theta, \theta^{\prime} \in\{ \pm 1\}$.

For $p=2$, one takes $\theta \in \mathbf{Z}_{2}^{*} /\left(\mathbf{Z}_{2}^{*}\right)^{2}$, there are also two other kind of generators and further relations; cf. [Nik8], Prop. 1.8.1 and 1.8.2. 
Definition 2.B An even lattice is a free $\mathbf{Z}$-module of finite rank together with a non-

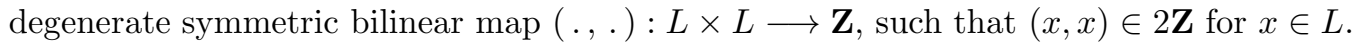

The map $(.,$.$) can be linearly extended to L \otimes R$ for any ring $R \supset \mathbf{Z}$. Every lattice $L$ defines a quadratic space $(A, q)$ by letting $A=L^{*} / L$, where $L^{*}=\{x \in L \otimes \mathbf{Q} \mid(x, y) \in$ $\mathbf{Z}$ for all $y \in L\}$ is the dual lattice, and $q: L^{*} / L \longrightarrow \mathbf{Q} / 2 \mathbf{Z}$ is the quadratic form $x$ $(\bmod L) \mapsto q(x)=(x, x) \quad(\bmod 2 \mathbf{Z})$, called the discriminant form. The signature $\operatorname{sign}(L)$ of $L$ is the pair $\left(n_{+}, n_{-}\right)$, where $n_{+}$resp. $n_{-}$are the maximal dimension of a positive resp. negative definite subspace of $L \otimes \mathbf{R}$. By Sylvester's Law of inertia, $n_{+}+n_{-}=\operatorname{rk}(L)$, the rank of $L$.

Definition 2.C The genus of $L$ is the collection of the local lattices $L \otimes \mathbf{Z}_{p}, p$ a prime number, including $L \otimes \mathbf{R}$ for $p=\infty$.

It follows easily that the genus of $L$ determines $\left(L^{*} / L, q\right)$ and $\operatorname{sign}(L)$. Also, the converse is true:

Theorem 2.2 (cf. Nik80], Cor. 1.9.4) The discriminant form $\left(L^{*} / L, q\right)$ and the signature $\operatorname{sign}(L)$ determine the genus of $L$.

Not every pair consisting of a finite quadratic space $(A, q)$ and a pair $\left(n_{+}, n_{-}\right)$of nonnegative integers can be realized as the genus of a lattice. First, there is a condition modulo 8:

Theorem 2.3 (Milgram) For a lattice $L$ with discriminant form $\left(L^{*} / L, q\right)$ and signature $\left(n_{+}, n_{-}\right)$, one has

$$
\frac{1}{\sqrt{\left|L^{*} / L\right|}} \sum_{x \in L^{*} / L} e^{\pi i q(x)}=e^{2 \pi i\left(n_{+}-n_{-}\right) / 8}
$$

The next result shows that for ranks large enough this is the only condition that the quadratic space and the signature have to satisfy.

Theorem 2.4 An even lattice $L$ with discriminant form $(A, q)$ and signature $\left(n_{+}, n_{-}\right)$exists if condition (1) is satisfied and $n_{+}+n_{-}>\operatorname{rank}(A)$.

Note that $n_{+}+n_{-} \geq \operatorname{rank}(A)$ is clearly a necessary condition. For $n_{+}+n_{-}=\operatorname{rank}(A)$, one also has a precise but more complicated condition for the existence of a lattice; see [Nik80], Th. 1.10.1.

\section{Theorem 2.5 (Finiteness of the class number) The set}

$$
\operatorname{gen}(L):=\{\text { Isomorphy classes of lattices with the same genus as } L\}
$$

is finite.

The proof uses the result that there are only finitely many lattices $L$ of fixed discriminant $\left|L^{*} / L\right|$; see for example Cas78, Ch. 9, Th. 1.1.

Much more precise information about the number of lattices in a genus is known. We restrict ourselves to positive definite lattices, i.e., lattices of rank $n=n_{+}$. 


\section{Theorem 2.6 (Mass formula)}

$$
\sum_{M \in \operatorname{gen}(L)} \frac{1}{|\operatorname{Aut}(M)|}=2 \cdot \alpha_{\infty} \times \prod_{p=2,3,5, \ldots} \alpha_{p},
$$

where $\alpha_{\infty}=2 \pi^{-n(n+1) / 4}\left(\prod_{j=1}^{n} \Gamma(j / 2)\right) \cdot\left|L^{*} / L\right|^{(n+1) / 2}$ and the $\alpha_{p}$ 's can be computed explicitly and depend only on $n$ and the p-component $\left(\left(L^{*} / L\right)_{p}, q_{p}\right)$.

This result was obtained independently by H. J. S. Smith Smi1867 and H. Minkowski Min1884.

The number of embeddings of lattices $K$ of rank $g \leq n$ into $L$ for all $K$ can be encoded in the Siegel Theta series $\Theta_{L}^{(g)}(Z)$ of genus $g$, a function on the Siegel upper half plane $\mathbf{H}_{g}=\left\{Z \in \operatorname{Mat}(g, \mathbf{C}) \mid Z=Z^{t}, \operatorname{Im}(Z)>0\right\}$ of genus $g$ :

$$
\Theta_{L}^{(g)}(Z)=\sum_{v_{1}, \ldots, v_{g} \in L} e^{2 \pi i \operatorname{tr}\left(\left(v_{1}, \ldots, v_{g}\right)\left(v_{1}, \ldots, v_{g}\right)^{t} Z\right)}
$$

It is a modular form of weight $n / 2$ for a congruence subgroup $\Gamma$ of $\operatorname{Sp}(g, \mathbf{Z})$ acting on $\mathbf{H}_{g}$.

Theorem 2.7 (Siegel)

$$
\left(\sum_{M \in \operatorname{gen}(L)} \frac{\Theta_{M}^{(g)}(Z)}{|\operatorname{Aut}(M)|}\right) \cdot\left(\sum_{M \in \operatorname{gen}(L)} \frac{1}{|\operatorname{Aut}(M)|}\right)^{-1}=E^{(g)}(Z),
$$

where $E^{(g)}(Z)$ is an explicitly given Eisenstein series for $\Gamma$, depending only on the genus of $L$.

This theorem was first proven by C. L. Siegel Sie35] and reformulated in an adelic picture by A. Weil, cf. Wei65, see also Kud84 for a general setting.

There is a slightly finer invariant for lattices, namely M. Eichler's spinor genus, cf. Cas78]. The set of lattices in a genus can be decomposed into $2^{r}$ spinor genera, also very often $r=0$. The sum $\sum_{M} \frac{1}{|\operatorname{Aut}(M)|}$ over all lattices $M$ in the same spinor genus as a positive definite lattice $L$ is for all spinor genera in the genus of $L$ the same if $\operatorname{rank}(L) \geq 3$.

For indefinite lattices of rank at least 3 , there is only one lattice in each spinor genus. This is one way to see that there is only one even unimodular (i.e., of discriminant 1 ) lattice $I I_{r, s}$ of signature $(r, s)$ if $\min (r, s) \geq 1$. Furthermore, one can deduce that two lattices $L$ and $M$ are in the same genus if and only if $L \oplus I I_{1,1}$ and $M \oplus I I_{1,1}$ are isomorphic.

There is also the concept of rational equivalence. Two lattices $L$ and $M$ are called rational equivalent if the rational quadratic spaces $L \otimes \mathbf{Q}$ and $M \otimes \mathbf{Q}$ are isomorphic. An equivalent more geometric formulation is to say that both $L$ and $M$ have an isomorphic sublattice $K$ of finite index. From the Hasse principle, it follows that $L$ and $M$ are rational equivalent if and only if all the local quadratic spaces $L \otimes \mathbf{Q}_{p}$ and $M \otimes \mathbf{Q}_{p}$, for $p$ a prime or $\infty$, are isomorphic. In particular, lattices in the same genus are rational equivalent.

Finally, we remark that the results explained in this section hold similarly for non-even integral lattices. 


\section{Genera of a vertex operator algebras}

In this section, we explain how the concepts from the arithmetic of quadratic forms explained in the last section can, at least partially, be generalized to vertex operator algebras.

A good starting point for the description of three-dimensional topological quantum field theories are modular braided tensor categories TTur94. With the help of the Kirby calculus [Kir78], one can use them to define invariants of 3-manifolds, cf. [RT91]. We will give the basic definitions, for details see [Tur94].

Definition 3.A A monoidal category is a category $\mathcal{C}$ together with a functorial associative tensor product $\otimes: \mathcal{C} \times \mathcal{C} \longrightarrow \mathcal{C}$ and a neutral object 1 for $\otimes$. We assume that the tensor category is strict, so for all objects $U, V, W$ of $\mathcal{C}$ the products $(U \otimes V) \otimes W$ and $U \otimes(V \otimes W)$ are identical and not just isomorphic objects.

A ribbon category is a monoidal category together with:

(i) Functorial isomorphisms $R_{U, V}: U \otimes V \longrightarrow V \otimes U$ (the braiding) such that

$$
R_{U, V \otimes W}=\left(\operatorname{id}_{V} \otimes R_{U, W}\right)\left(R_{U, V} \otimes \operatorname{id}_{W}\right) \text { and } R_{U \otimes V, W}=\left(R_{U, W} \otimes \operatorname{id}_{V}\right)\left(\operatorname{id}_{U} \otimes R_{V, W}\right) .
$$

(ii) Functorial isomorphisms $\theta_{V}: V \longrightarrow V$ (the twist) such that

$$
\theta_{U \otimes V}=R_{V, U}\left(\theta_{V} \otimes \theta_{U}\right) R_{U, V}
$$

(iii) A triple $(*, d, b)$, which associates to any $V$ a dual object $V^{*}$ and morphisms $d$ : $V \otimes V^{*} \longrightarrow \mathbf{1}$ (the evaluation) and $b: \mathbf{1} \longrightarrow V \otimes V^{*}$ (the coevaluation), such that

$$
\begin{gathered}
\left(d_{V} \otimes \mathrm{id}_{V^{*}}\right)\left(\mathrm{id}_{V^{*}} \otimes b_{V}\right)=\mathrm{id}_{V^{*}} \text { and }\left(\mathrm{id}_{V^{*}} \otimes d_{V}\right)\left(b_{V} \otimes \mathrm{id}_{V}\right)=\mathrm{id}_{V}, \\
\left(\theta_{V} \otimes \mathrm{id}_{V^{*}}\right) b_{V}=\left(\mathrm{id}_{V} \otimes \theta_{V^{*}}\right) b_{V} .
\end{gathered}
$$

These axioms can be visualized by labeled bands in $\mathbf{R}^{3}$. In a ribbon category, one can define for any endomorphism $f: V \longrightarrow V$ the trace of $f$ as an element of $\operatorname{End}(\mathbf{1})$ :

$$
\operatorname{tr}(f)=d_{V} R_{V, V^{*}}\left(\theta_{V} f \otimes \operatorname{id}_{V^{*}}\right) b_{V} .
$$

The quantum dimension $\operatorname{dim}(V)$ of $V$ is the trace of the identity morphism of $V$.

A modular braided tensor category (over $\mathbf{C}$ ), or modular category for short, is a ribbon category which has also the structure of an abelian category over $\mathbf{C}$ such that:

(i) the tensor product is $\mathbf{C}$-linear and $\operatorname{End}(\mathbf{1})=\mathbf{C}$;

(ii) every object is a direct sum of a finite set of simple objects;

(iii) the isomorphism classes of simple objects form a finite set $\left\{V_{i}\right\}_{i \in I}$ and an object $V$ is simple precisely if $\operatorname{End}(V)=\mathbf{C}$;

(iv) the matrix $\left(\operatorname{tr}\left(R_{V_{j}, V_{i}} R_{V_{i}, V_{j}}\right)\right)_{i, j \in I}$ is invertible. 
We call the number $D=\sum_{i \in I}\left(\operatorname{dim}\left(V_{i}\right)\right)^{2}$ the discriminant. In Tur94, a square root $\sqrt{D}$ is called a rank. The $S$-matrix is defined as the matrix $S^{\mathrm{TOP}}=\frac{1}{\sqrt{D}}\left(\operatorname{tr}\left(R_{V_{j}, V_{i}} R_{V_{i}, V_{j}}\right)\right)_{i, j \in I}$ We also need $\gamma=\left(\sum_{i \in I} \theta_{i}\left(\operatorname{dim}\left(V_{i}\right)\right)^{2} / \sqrt{D}\right)^{1 / 3}$. Here, the numbers $\left\{\theta_{i}\right\}_{i \in I}$ are defined by $\theta_{V_{i}}=\theta_{i} \cdot \operatorname{id}_{V_{i}}$. The $T$-matrix is the diagonal matrix $T^{\mathrm{TOP}}$ with the $\gamma^{-1} \cdot \theta_{i}, i \in I$, on the diagonal. The $S$ - and $T$-matrix satisfy the relations $(S T)^{3}=S^{2}, S^{2} T=T S^{2}$ and $S^{4}=1$. These are the same relations as for the generators $s=\left(\begin{array}{rr}0 & 1 \\ -1 & 0\end{array}\right)$ and $t=\left(\begin{array}{ll}1 & 1 \\ 0 & 1\end{array}\right)$ of $\mathrm{SL}_{2}(\mathbf{Z})$, the mapping class group of a genus 1 surface. Therefore the $S$ - and $T$-matrix define a complex representation $\rho$ of $\mathrm{SL}_{2}(\mathbf{Z})$ of dimension $|I|$. If we omit the factor $\gamma^{-1}$ in the definition of the $T$-matrix, we obtain a more natural projective representation of $\mathrm{SL}_{2}(\mathbf{Z})$, cf. Tur94, II.3.9. In the context of modular categories, one can prove that the $\theta_{i}$ and $\gamma$ are roots of unity; cf. Vaf88, AM88, DML2000, Boj2001.

Finally, there is the notation of a unitary modular braided tensor category where one has natural maps

$$
\text { - } \operatorname{Hom}(V, W) \longrightarrow \operatorname{Hom}(W, V)
$$

identical to complex conjugation for $V=W=V_{i}, i \in I$, such that $\operatorname{tr}(f \bar{f}) \geq 0$ for all $f \in \operatorname{Hom}(V, W)$.

Two modular categories are called equivalent, if there is a functorial isomorphism between them, carrying over all structures.

One can also consider nonstrict monoidal categories and define modular categories by using them. Every monoidal category is equivalent to a strict one by MacLane's coherence theorem and this is also true for modular braided tensor categories, cf. [Tur94], XI.1.4. The categories arising from conformal field theory are in general not strict.

We formulate two versions of the Verlinde formula which are theorems in the context of modular categories. First, let $\left(N_{i j}^{k}\right)^{\text {TOP }}=\operatorname{dim} \operatorname{Hom}\left(V_{i} \otimes V_{j}, V_{k}\right)$ be the structure constants of the fusion algebra defined on the free vector space $\mathbf{C}[I]$ by the product $i \times j=\sum_{k \in I}\left(N_{i j}^{k}\right)^{\mathrm{TOP}} k$. Then one has

$$
\operatorname{dim} \operatorname{Hom}\left(V_{i} \otimes V_{j}, V_{k}\right)=\sum_{l \in I} \frac{S_{i l} S_{j l} S_{k l^{*}}}{S_{0 l}},
$$

where $i^{*} \in I$ is the label defined by $V_{i^{*}} \cong V_{i}^{*}$ and $0 \in I$ is the label for $V_{0} \cong \mathbf{1}$. Secondly, let $N_{i_{1}, \ldots, i_{n}}^{\mathrm{TOP}}(g)$ be the dimension of the vector space associated by the $3 d$-TQFT to a genus $g$ surface decorated with labels $i_{1}, \ldots, i_{n}$. Then one has

$$
N_{i_{1}, \ldots, i_{n}}^{\mathrm{TOP}}(g)=D^{g-1} \sum_{j \in I}\left(\left(\operatorname{dim}\left(V_{j}\right)\right)^{2-2 g-n} \prod_{s=1}^{n} S_{i_{s}, j}\right) .
$$

We mention three important classes of examples of modular categories:

1) The modular category $\mathcal{C}(A, q)$ associated to a finite quadratic space $(A, q)$, see [Tur94], I.1.7.2 and II.1.7.2. We have $q: A \longrightarrow \mathbf{Q} / 2 \mathbf{Z} \subset U(1) \subset \mathbf{C}^{*}, q(x)=\varphi(x) c(x, x)$, where the maps $c: A \times A \longrightarrow \mathbf{C}^{*}, \varphi:{ }_{2} A \longrightarrow \mathbf{C}^{*}\left({ }_{2} A\right.$ denotes the 2-torsion subgroup of $\left.A\right)$ used in [Tur94] are up to equivalence identical to the map $q$, see [FK93], Sec. 7.5. Since the fusion algebra of $\mathcal{C}(A, q)$ is just the group ring $\mathbf{C}[A]$ of the abelian group $A$, we call such a modular category abelian. Any modular category with such an abelian group ring $\mathbf{C}[A]$ as fusion algebra arises in such a way, see [FK93], Ch. 7. 
2) The Chern-Simons theory associated to a simple Lie group $G$ and positive integral level $k$. This modular categories can be constructed using quantum groups, cf. Dri87, RT91].

3) Discrete Chern-Simons theories constructed from a finite group $G$ and a class $c \in H^{3}(G, \mathbf{C})$ DW90, FQ93.

Problem 3.1 Find a good description of the set of equivalence classes of modular braided tensor categories over $\mathbf{C}$.

For two such categories $\mathcal{C}$ and $\mathcal{D}$, it is easy to define a product category $\mathcal{C} \times \mathcal{D}$, such that its simple objects are the products of the simple objects of the factors. One can decompose a category into indecomposable pieces $\mathcal{C}_{s}$ under the product: $\mathcal{C}=\mathcal{C}_{1} \times \mathcal{C}_{2} \times \ldots \times \mathcal{C}_{r}$. An additional problem compared to the situation for quadratic spaces is that it is not possible to decompose $\mathcal{C}$ into a product of local pieces $\mathcal{C}_{p}$ for different primes $p$ such that, for example, the numbers $\theta_{i}$ for $\mathcal{C}_{p}$ are $p$-th root of unity.

A conjecture for modular braided tensor categories (also usually formulated for rational VOAs), says that the above introduced representation $\rho: \mathrm{SL}_{2}(\mathbf{Z}) \longrightarrow \operatorname{End}(\mathbf{C}[I])$ has a congruence subgroup as kernel or, more precisely, $\operatorname{ker}(\rho) \supset \Gamma(N)$ where $N$ is the smallest natural number such that $T^{N}=1$. Away from the prime $p=2$, this has been proven by Coste and Gannon [CG99. P. Bantay Ban2001 has recently reduced the problem to a property of permutation orbifolds. Assuming this conjecture, Eholzer [Eho95] has classified the indecomposable modular categories (or at least the fusion algebras together with the $T$-matrix and $\gamma$ ) with $|I| \leq 4$ using the representation theory of $\mathrm{SL}_{2}(\mathbf{Z} / N \mathbf{Z})$. A lot of work has been done on the classification of fusion algebras and also compatible tensor categories, cf. for example [FK93, Fuc94, MN2001].

For the purpose of this paper, the following definition of a restricted class of vertex algebras seems to be most adequate.

Definition 3.B A vertex operator algebra of central charge $c$ is a graded complex vector space $V=\bigoplus_{n \in \mathbf{Z}_{>0}} V_{n}$ with $\operatorname{dim} V_{n}<\infty$ together with a linear map $Y(., z): V \longrightarrow$ $\operatorname{End}(V)\left[\left[z, z^{-1}\right]\right]$ (the state field correspondence) such that for $v \in V_{m}$ the coefficients $v_{n}$ of $Y(v, z)=\sum_{n \in \mathbf{Z}} v_{n} z^{-n-1}$ are of degree $m-n-1$ and $v_{n} u=0$ for fixed $u \in V$ and $n$ large enough, and there are two distinguished elements $\mathbf{1} \in V_{0} \cong \mathbf{C}$ (the vacuum) and $\omega \in V_{2}$ (the Virasoro element) subject to the following axioms:

- $Y(\mathbf{1}, z)=\mathrm{id}_{V}$ and for $v \in V$, one has $v_{n} \mathbf{1}=0$ for $n \geq 0, v_{-1} \mathbf{1}=v$.

- For $u, v \in V$, there is a $N \in \mathbf{Z}_{\geq 0}$ with $(z-w)^{N} Y(u, z) Y(v, w)=$ $(z-w)^{N} Y(v, w) Y(u, z)$ in $\operatorname{End}(V)\left[\left[z, z^{-1}, w, w^{-1}\right]\right]$.

- The coefficients of $Y(\omega, z)=\sum_{n \in \mathbf{Z}} L_{n} z^{-n-2}$ define a representation of the Virasoro algebra of central charge $c$ : $\left[L_{m}, L_{n}\right]=L_{m+n}+\frac{m^{3}-m}{12} \delta_{m+n, 0} \cdot c \cdot \operatorname{id}_{V}$ and for $v \in V$, one has $Y\left(L_{-1} v, z\right)=\frac{d}{d z} Y(v, z)$.

These axioms can be distilled from the general axioms of axiomatic quantum field theory applied to the case of Riemannian surfaces as the space time. To obtain a more geometric formulation as indicated in the introduction from a VOA, further conditions have to be satisfied. For the terms we use below and for details, we refer to the literature: 
There is the notion of modules and intertwining spaces $\left(\begin{array}{c}M_{3} \\ M_{1}, M_{2}\end{array}\right)$ for a VOA, see FHL93. A VOA is usually called rational, if there are only finitely many nonisomorphic irreducible modules and any module can be decomposed into a finite direct sum of irreducible ones. (The first condition can be deduced from the second, cf. [DML98].) The conformal weight $h$ of a module is its smallest $L_{0}$-eigenvalue. Let $\left\{M_{j}\right\}_{j \in I}$ be a complete set of nonisomorphic irreducible modules. The $T$-matrix is defined as the diagonal matrix $T^{\mathrm{VOA}}$ with the numbers $e^{-2 \pi i c / 24} \cdot e^{2 \pi i h_{j}}, j \in I$, on the diagonal. Zhu has shown Zhu9d that under some conditions on the rational VOA, there is a representation of $\mathrm{SL}_{2}(\mathbf{Z})$ on some space of genus 1 correlation functions. The generator $s$ of $\mathrm{SL}_{2}(\mathbf{Z})$ defines the matrix $S^{\mathrm{VOA}}$. Using the tensor product theory for modules of VOAs, Huang and Lepowsky associated a (nonstrict) braided tensor category to a suitable class of VOAs, see HL94 and the references in HL99]. Finally, there is a definition for the dimension $N_{i_{1}, \ldots, i_{n}}^{\mathrm{VOA}}(g)$ of the space of vacua on the genus $g$ surface with labels $i_{1}, \ldots, i_{n}$ by Zhu Zhu94.

I assume further that the VOA is unitary, this means the VOA can be defined over the real numbers and the natural symmetric form on it is positive definite, although this will exclude many interesting examples. Also, the VOA should be simple, i.e., $V$ is irreducible as a module over itself.

We like to use the above mentioned results and some other properties believed to be true for interesting VOAs. We therefore make the following assumption on the VOAs considered:

Assumption 3.2 The VOA $V$ satisfies the conditions needed in the construction of the braided tensor category on the category of modules as in [HL94 and also satisfies the conditions used in [Zhu9d, Zhu94].

Furthermore, the braided tensor category of $V$-modules has the structure of a modular braided tensor category $\mathcal{C}(V)$ such that

$$
\begin{aligned}
e^{2 \pi i c / 24} \cdot T^{\mathrm{VOA}} & =\gamma \cdot T^{\mathrm{TOP}}, \\
S^{\mathrm{VOA}} & =S^{\mathrm{TOP}}, \\
\operatorname{dim}\left(\begin{array}{c}
M_{k} \\
M_{i}, M_{j}
\end{array}\right) & =\operatorname{dim} \operatorname{Hom}\left(V_{i} \otimes V_{j}, V_{k}\right), \quad \text { for } i, j, k \in I, \\
N_{i_{1}, \ldots, i_{n}}^{\mathrm{VOA}}(g) & =N_{i_{1}, \ldots, i_{n}}^{\mathrm{TOP}}(g), \quad \text { for } i_{1}, \ldots, i_{n} \in I, g \geq 0 .
\end{aligned}
$$

One problem is certainly to find simple sufficient conditions on the VOA so that the assumption becomes a theorem. Sometimes other conditions in the definition of rationality are added. Assuming that Assumption 3.2 is true would directly imply that the central charge and conformal weights are rational numbers.

Property (iv) follows from (iii) if one can prove the sewing relations

$$
\sum_{k \in I} N_{i_{1}, \ldots, i_{n}, k}^{\mathrm{VOA}}(g) \cdot N_{k^{*}, j_{1}, \ldots, j_{m}}^{\mathrm{VOA}}(h)=N_{i_{1}, \ldots, i_{n}, j_{1}, \ldots, j_{m}}^{\mathrm{VOA}}(g+h)
$$

and

$$
\sum_{k \in I} N_{i_{1}, \ldots, i_{n}, k, k^{*}}^{\mathrm{VOA}}(g)=N_{i_{1}, \ldots, i_{n}}^{\mathrm{VOA}}(g+1) .
$$

For important examples of VOAs, at least parts of the assumption are known to be true; for details we refer to the references:

1) VOAs defining an abelian intertwining algebra, cf. DL93, Ros01. 
2) Wess-Zumino-Witten models, i.e., the VOAs defined on the highest weight representations of level $k$ for an affine Kac-Moody Lie algebra Lie $(G)$; cf. TUY89, KL91, EZ92, Fal94, Fin96, HL99.

3) Holomorphic orbifolds; cf. [DVVV89, Hua96a, DM96.

4) Minimal models; cf. Hua96b, HM99, HM2002].

One could try to generalize the above mentioned results for rational vertex algebras. In their definition one allows $\mathbf{Z}$-graded vector spaces $V$ and the pieces $V_{n}$ are not assumed to be finite-dimensional. They should be considered as the analog of indefinite even lattices. For a vertex algebra $V$ of central charge $c$, one can define the number $c_{+}$as the largest central charge of a subVOA $W$ of $V, c_{-}$as $c-c_{+}$and denote the pair $\left(c_{+}, c_{-}\right)$the signature of $V$.

For the rest of this section, we consider only VOAs for which Assumption 3.2 is satisfied.

Definition 3.C The genus of a rational VOA $V$ of central charge $c$ is defined as the pair consisting of the associated modular braided tensor category $\mathcal{C}(V)$ and the central charge $c$.

It is not known much about the set of central charges which can occur. From the discussion of modular categories, we know that $c$ has to be rational. The theory of unitary highest weight representations of the Virasoro algebra shows that $c$ belongs to the minimal series $c=1-\frac{6}{n(n+1)}, n=3,4, \ldots$, for $c<1$.

There is the following analogue of Milgrams theorem for modular braided tensor categories.

Theorem 3.3 (cf. Tur94) For a rational VOA $V$ with associated modular category $\mathcal{C}(V)$ and central charge $c$, one has

$$
\frac{1}{\sqrt{\sum_{i \in I} \operatorname{dim}\left(V_{i}\right)^{2}}} \sum_{j \in I} e^{2 \pi i h_{j}} \operatorname{dim}\left(V_{j}\right)^{2}=e^{2 \pi i c / 8} .
$$

Proof: The result is an immediate consequence of Assumption 3.2 and the existence of the $\mathrm{SL}_{2}(\mathbf{Z})$-representations. We have $\left(S^{\mathrm{VOA}} T^{\mathrm{VOA}}\right)^{3}=\left(S^{\mathrm{VOA}}\right)^{2}$ and $\left(S^{\mathrm{TOP}} T^{\mathrm{TOP}}\right)^{3}=$ $\left(S^{\mathrm{TOP}}\right)^{2}$ because of the $\mathrm{SL}_{2}(\mathbf{Z})$-representations. Using properties (i) and (ii) in Assumption 3.2, we get $e^{2 \pi i c / 8}=\gamma^{3}$.

Problem 3.4 Let $\mathcal{C}$ be a modular braided tensor category. Does there exist rational VOAs $V$ of central charge $c$ with $\mathcal{C}(V)=\mathcal{C}$ if condition (6) is satisfied and $c$ is large enough?

What are the possible values of $c$ for abelian $\mathcal{C} \cong \mathcal{C}(A, q)$ ? For VOAs there are more central charges $c$ possible than one can realize by lattice VOAs. The bound $c \geq \operatorname{rank}(A)$ does not hold in general: The fixpoint subVOA $V_{\sqrt{2} E_{8}}^{\tau}$ of the lattice VOA $V_{\sqrt{2} E_{8}}$ for the involution $\tau$ lifted from the reflection at the origin of $\sqrt{2} E_{8}$ (cf. Gri98]) has central charge 8, but $\mathcal{C}\left(V_{\sqrt{2} E_{8}}^{\tau}\right) \cong \mathcal{C}\left(\mathbf{F}_{2}^{10}, q\right)$, where $q$ is isomorphic to 5 copies of the hyperbolic plane.

Conjecture 3.5 The set

$$
\operatorname{gen}(V):=\{\text { Isomorphy classes of VOAs with the same genus as } V\}
$$

is finite. 
Example: Let $\mathcal{C}$ be the trivial modular category, i.e., there is only one simple object. The corresponding VOAs are called self-dual or holomorphic. By Theorem 3.3, the central charge $c$ has to be divisible by 8 . An interesting case is $c=24$. Schellekens has found a list of 71 candidates for the corresponding genus [Sch93]. For the 24 lattice VOAs constructed from the genus of the positive definite even unimodular lattices in dimension 24, one has an existence and uniqueness theorem. $24-9$ other VOAs are completely constructed as $\mathbf{Z}_{2}$-orbifolds, cf. DGM90, DGM90b. One of them is the Moonshine module $V^{\natural}$, cf. Bor86, FLM88. The fact that $V^{\natural}$ belongs to the genus was proven in Don94. The uniqueness conjecture FLM88] that $V^{\natural}$ is the only VOA $V$ in the genus with $\operatorname{dim} V_{1}=0$ is still open. For the VOA with $V_{A_{1,2}}^{\otimes 16}$ as affine Kac-Moody subVOA, we can show uniqueness, cf. [GH2001 and next section.

Conformal field theory suggests that for $g \geq 1$ it may be possible to define a (possibly vector valued) genus $g$ partition function $\chi_{V}^{(g)}$ on the genus $g$ Teichmüller space $\mathcal{T}_{g}$ with automorphic properties for the mapping class group of a genus $g$ surface. For $g=1$ this follows from Zhu's work Zhu90.

Problem 3.6 Are there good Mass formulas for the genus of a VOA of the form

$$
\sum_{W \in \operatorname{gen}(V)} \frac{1}{|\operatorname{Aut}(W)|} \text { and } \sum_{W \in \operatorname{gen}(V)} \frac{\chi_{W}^{(g)}(Z)}{|\operatorname{Aut}(W)|} ?
$$

One problem is to find a good definition for $|\operatorname{Aut}(V)|$ if $\operatorname{Aut}(V)$ is infinite; one could try to take the Tamagawa measure if $\operatorname{Aut}(V)$ is a reductive Lie group defined over $\mathbf{Q}$ by using VOAs defined over Q. For a kind of relative Mass formula of this type involving a nonabelian extension problem, see Theorem 4.5 in the next section.

The definition of the genus of a VOA was modeled along the notation of the genus of an even lattice $L$ such that the following diagram is commutative

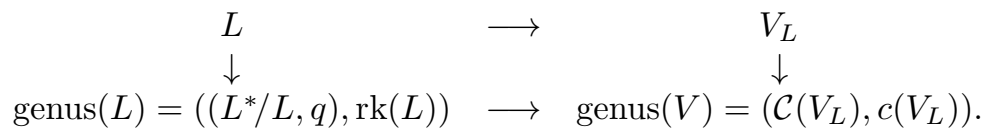

The upper horizontal arrow is the lattice construction of VOAs Bor86], the left down arrow gives the genus of a lattice via the discriminant form, the right down arrow comes from the abelian intertwining structure on the modules of $V_{L}$ DL93 and the lower horizontal arrow is the map associating the abelian modular category to a finite quadratic space as explained as an example above.

Regarding genera of vertex algebras, a structure theory seems to be more complicated than for indefinite lattices: The vertex algebras $V^{\natural} \otimes V_{I I_{1,1}}$ and $V_{\Lambda} \otimes V_{I I} \cong V_{I I_{25,1}}$ (here, $\Lambda$ denotes the Leech lattice) are not isomorphic since both define in a uniform way different generalized Kac-Moody Lie algebras, namely the Monster Bor92] and the fake Monster Lie algebra Bor90]. (They are different because all Cartan subalgebras of a generalized KacMoody Lie algebra are conjugate by inner automorphisms as shown by U. Ray Ray01.) But both should be self-dual vertex algebras of signature $(25,1)$. Another such example is constructed in HS2001.

A natural generalization of the notion of rational equivalence from lattices to VOAs would be as follows: We take the smallest equivalence relation such that two rational VOAs $V$ and $W$ of central charge $c$ are equivalent if both have isomorphic rational subVOAs with 
the same Virasoro element. This means that there exist VOAs $U_{0}=V, U_{1}, \ldots, U_{r}=W$ and VOAs $S_{0}, \ldots, S_{r-1}$ such that $S_{i}$ is a common subVOA of $U_{i}$ and $U_{i+1}$ for $i=0, \ldots, r-1$. Just to say there exists a common isomorphic subVOA wouldn't give an equivalence relation: take for $V$ and $W$ the fixpoint VOAs $V=U^{G}$ and $W=U^{G^{\prime}}$ where $U$ is the $A_{1} \cong \frac{1}{\sqrt{2}} \mathbf{Z}$ lattice VOA and $G$ and $G^{\prime}$ are finite subgroups of SU(2) which can't be conjugated into the same larger finite subgroup of $\mathrm{SU}(2)$ (like the binary icosahedral and the binary tetrahedral group).

Also, one can generalize the discussion of this section to vertex operator super algebras (SVOAs). The central charge $c$ of a self-dual SVOA has to be half-integral [Höh95], Th. 2.2.2. For an interesting example with $c=23 \frac{1}{2}$ and the classification for small $c$ see also Höh95.

\section{Extension problems}

In this section, we explain how extension problems for suitable VOAs can be formulated in terms of the associated modular braided tensor category and sometimes solved by this methods.

For even lattices, all overlattices can easily be described; cf. [Nik80]:

Theorem 4.1 (Lattice extensions) (a) The even overlattices $K$ of an even lattice $L$ are in one-to-one correspondence to the isotropic subspaces $C$ of the associated quadratic space $\left(L^{*} / L, q\right)$.

(b) The quadratic space for $K$ is given by the pair $\left(C^{\perp} / C,\left(q \mid C^{\perp}\right) / C\right)$.

(c) Two such extensions give isomorphic lattices $K$ exactly if the corresponding subspaces can be transformed into each other by an isometry of $\left(L^{*} / L, q\right)$ induced from an automorphism of $L$.

For integral lattices, one has to take subspaces $C$ on which the induced bilinear form $b_{L}: C \times C \longrightarrow \mathbf{C}$ is trivial.

Our aim is to present similar results for extensions of VOAs. Let $V$ be a rational VOA. We call a VOA $\left(W, Y_{W}\right)$ an extension of $V$ if it contains a subVOA isomorphic to $V$ and has the same vacuum and Virasoro element as $V$. We have $W \cong \bigoplus_{i \in I} n_{i} M_{i}$ for a decomposition of $W$ into irreducible $V$-modules $M_{i}$, where $\left\{M_{i}\right\}_{i \in I}$ is a complete set of irreducible modules of $V$ and the nonnegative integers $n_{i}$ are the multiplicities of $M_{i}$ in $W$. Also, we assume that we have fixed an embedding $\iota_{W}: V \longrightarrow W$.

We call two extensions $\left(W, Y_{W}\right)$ and $\left(W^{\prime}, Y_{W^{\prime}}\right)$ isomorphic if there is an VOAisomorphism $\sigma: W \longrightarrow W^{\prime}$ and an automorphism $\tau$ of $V$ such that $\sigma \circ \iota_{W}=\iota_{W^{\prime}} \circ \tau$. For $\left(W, Y_{W}\right)=\left(W^{\prime}, Y_{W^{\prime}}\right)$ and $\iota_{W}=\iota_{W^{\prime}}$, we can define in this way the automorphism group $\operatorname{Aut}_{V}(W)$ of the extension $\left(W, Y_{W}\right)$.

Part (a) of Theorem 4.1 implies that the overlattices of a lattice can be determined from the structure of its discriminant form. For VOAs we have as an analogous result:

Theorem 4.2 (see [Höh97], p. 615) The VOA-extensions $W$ of a rational VOA $V$ satisfying Assumption 3.9 can be determined completely in terms of the associated modular braided tensor category $\mathcal{C}(V)$. 
Proof: Fix a $V$-module isomorphism $W \cong \bigoplus_{i \in I} n_{i} M_{i}$ extending $\iota_{W}$. The vertex operator $Y_{W}$ is an element of

$$
\bigoplus_{i, j, k \in I} \operatorname{Hom}\left(\mathbf{C}^{n_{i}} \otimes \mathbf{C}^{n_{j}}, \mathbf{C}^{n_{k}}\right) \otimes\left(\begin{array}{c}
M_{k} \\
M_{i} M_{j}
\end{array}\right)_{V}
$$

For $\left(W, Y_{W}\right)$ to be a VOA-extension of $V$, the modules $M_{i}$ with $n_{i}>0$ have to be of integral conformal weight and $Y_{W}$ has to satisfy the commutativity axiom. Both conditions are completely controlled by the twist and the braiding of the associated modular braided tensor category.

Similarly, one can see that the structure of the intertwining algebra of an extension is completely determined by $\mathcal{C}(V)$ and $Y_{W}$. W We expect that any rational VOA $V$ has only finitely many nonisomorphic extensions $\left(W, Y_{W}\right)$.

In the case that $\mathcal{C}(V)$ is abelian, i.e., $\mathcal{C}(V)=\mathcal{C}(A, q)$ for a finite quadratic space $(A, q)$, one could expect in generalization of Theorem 4.1 (b) that the VOA-extensions are determined by the isotropic subspaces of $A$. This is indeed the case. Recall that a simple module $M_{i}$ is called a simple current if for each simple module $M_{j}$ there is a another simple module $M_{j^{\prime}}$ such that $M_{i} \times M_{j}=M_{j^{\prime}}$ holds in the fusion algebra.

Theorem 4.3 (simple current extensions) Let $V$ be a rational VOA for which one has an abelian intertwining operator algebra structure on the direct sum of the simple currents. Let $C$ be a subgroup of the abelian group $A \subset I$ of labels of the simple currents for which the modules $M_{c}, c \in C$, have integral conformal weight. Then there exists a unique simple VOA-extension $\left(W, Y_{W}\right)$ of the form $W \cong \bigoplus_{c \in C} n_{c} M_{c}$ and one has $n_{c}=1$.

Proof: Existence: Choose a non-zero vector $\mathcal{Y}_{c, c^{\prime}} \in\left(\begin{array}{c}M_{c+c^{\prime}} \\ M_{c}, M_{c^{\prime}}\end{array}\right) \cong \mathbf{C}$ for $c, c^{\prime} \in C$, and define $Y_{W}=\bigoplus_{c, c^{\prime} \in C} \mathcal{Y}_{c, c^{\prime}}^{c+c}$. Recall Mac52 that the first cohomology groups $H^{i}\left(K(C, 2), \mathbf{C}^{*}\right)$ of the second Eilenberg-MacLane space $K(C, 2)$ (which is up to homotopy the unique CWcomplex with homotopy groups $\pi_{2}(K(C, 2))=C$, and $\pi_{i}(K(C, 2))=0$ for $\left.i \neq 2\right)$ can explicitly described by the following cochain complex:

$$
\begin{aligned}
& K^{5} \stackrel{\partial^{4}}{\longleftarrow} \quad K^{4} \quad \stackrel{\partial^{3}}{\longleftarrow} \quad K^{3} \quad \stackrel{\partial^{2}}{\longleftarrow} \quad K^{2} \quad \ldots \\
& \ldots \quad F: C \times C \times C \rightarrow \mathbf{C}^{*} \quad f: C \times C \rightarrow \mathbf{C}^{*} \quad \varphi: C \rightarrow \mathbf{C}^{*} \\
& H: C \times C \rightarrow \mathbf{C}
\end{aligned}
$$

The coboundary maps $\partial^{i}$ which we need are defined by $\partial^{2}(\varphi)=\widehat{\partial}^{2}(\varphi)$ and $\partial^{3}(f)=$ $\left(\widehat{\partial}^{3}(f), f\left(c, c^{\prime}\right) / f\left(c^{\prime}, c\right)\right)$, where $\widehat{\partial}^{i}$ denotes the usual group cohomology coboundary operator. Note that we can work with normalized cochains, i.e., cochains with $\varphi(0)=1, f(0, c)=$ $f(c, 0)=1$, respectively $F\left(0, c, c^{\prime}\right)=F\left(c, 0, c^{\prime}\right)=F\left(0, c, c^{\prime}\right)=1$ and $H(0, c)=H(c, 0)=1$ for $c, c^{\prime} \in C$. The commutativity and associativity properties for abelian intertwining algebras DL93 associate to $Y_{W}$ a cocycle $(F, H) \in K^{4}$. The operator $Y_{W}$ defines a VOA structure on $W$ if $(F, H)=(1,1)$. The cohomology group $H^{4}\left(K(C, 2), \mathbf{C}^{*}\right)$ can be identified

\footnotetext{
${ }^{1}$ During preparation of this manuscript, the paper [KO2001 by A. Kirillov, Jr. and V. Ostrik became available. The authors formulate the following precise analogues of part (a) and (b) of Theorem 4.1 (see Th. 6.2 of [KO2001]) using notation from Boj2001]: (a) The extensions $W$ of $V$ are in one-to-one correspondence to rigid $\mathcal{C}(V)$-algebras $A$ on which the twist $\theta$ is trivial. (b) The category of representations of $W$ can be identified with $\operatorname{Rep}^{0} A$.
} 
via $q^{\prime}(x)=H(x, x)$ with the space of quadratic forms $q^{\prime}$ on $C$, see Mac52, Th. 3. In our situation, we have $q^{\prime}=1$ since the modules $M_{c}, c \in C$, have integral conformal weight and $H(x, x)$ equals the exponential function applied to $2 \pi i$ times the conformal weight of $x$. Therefore, there exists a $f \in K^{3}$ with $\partial^{3}(f)=(F, G)$. Replacing $\mathcal{Y}_{c, c^{\prime}}$ by $f\left(c, c^{\prime}\right)^{-1} \mathcal{Y}_{c, c^{\prime}}$, we see that $\left(W, Y_{W}\right)$ is a VOA.

Uniqueness: Assume $\left(W, Y_{W}\right)$ is an extension. Similar as in the proof of Prop. 2.5 (4) of [DGH98], one gets $m_{c}=m_{c}^{\prime}$ for all $c, c^{\prime} \in C$. But $m_{0}=1$ since $M_{0}$ must have multiplicity one. One has $\mathcal{Y}_{c, c^{\prime}} \neq 0$ since by Proposition 11.9 of DL93 one has $Y_{W}(u, z) v \neq 0$ if $u$ and $v$ are not 0 . We can replace $\mathcal{Y}_{c, c^{\prime}}$ by $f\left(c, c^{\prime}\right) \mathcal{Y}_{c, c^{\prime}}$ where $f: C \times C \rightarrow \mathbf{C}^{*}$ is a 3-cocycle with $\partial^{3}(f)=(1,1)$. A $V$-module isomorphism of the space $W \cong \bigoplus_{c \in C} M_{c}$ defines for any irreducible $M_{c}, c \in C$, a scalar $\varphi(c) \in \mathbf{C}^{*}$, i.e., a two cocycle $\varphi \in K^{2}$, and $f$ is changed to $\partial^{2}(\varphi) f$. But from the universal coefficient theorem, $H^{3}\left(K(C, 2), \mathbf{C}^{*}\right)=\operatorname{Ext}\left(C, \mathbf{C}^{*}\right)$ and $\operatorname{Ext}\left(C, \mathbf{C}^{*}\right)=0$ since the $\mathbf{Z}$-module $\mathbf{C}^{*}$ is divisible. So, VOAs $\left(W, Y_{W}\right)$ for different choices of cocycles $f$ are isomorphic.

Remarks: In terms of the associated modular braided tensor category, the simple currents define a subcategory $\mathcal{A} \subset \mathcal{C}(V)$ which is abelian, i.e., $\mathcal{A} \cong \mathcal{C}(A, q)$ for a finite quadratic space $(A, q)$ (we allow here that $q$ is degenerate, i.e., $\mathcal{C}(A, q)$ has not to be modular): The space of quadratic forms on $A$ can be identified as before with the cohomology group $H^{4}\left(K(A, 2), \mathbf{C}^{*}\right)$ and one has $q=H(x, x)$. Furthermore, $q \mid C=1$ since the modules $M_{c}, c \in C$, have integral conformal weight, i.e, $C$ is an isotropic subspace of $(A, q)$.

The theorem completely solves the extension problem for VOAs $V$ for which on the direct sum of a complete set of nonisomorphic irreducible modules there is the structure of an abelian intertwining algebra. In this case, the modular braided tensor category $\mathcal{C}(W)$ of an extension $W$ are described as in Theorem 4.1 for lattices.

The structure of the modular braided tensor category $\mathcal{C}(W)$ for general simple current extensions was investigated in FSS96, where a conjecture for the set of irreducible $W$ modules and the corresponding $S$-matrix was made. This conjecture was proven in Müg00].

There is a natural action of the dual group $\hat{C}=\operatorname{Hom}(C, \mathbf{C}) \leq \operatorname{Aut}_{V}(W)$ on $W$.

In the context of braided tensor categories, the problem was also studied in [FK93], section 7.6.

Another special situation where an extension problem can be partially solved arises from finite group actions. On the VOA side, one has:

Theorem 4.4 (Galois correspondence [DM97, HMT99]) Let $W$ be a VOA and $G \subset$ $\operatorname{Aut}(W)$ be a finite subgroup of the automorphism group. Let $V=W^{G}$ be the fixpoint VOA. Then the extensions $U$ of $V$ which are contained in $W$ are in one-to-one correspondence to the subgroups of $G$, i.e., there is a subgroup $H \leq G$ such that $U=W^{H}$.

From the perspective of modular braided tensor categories, this situation was analyzed independently by Bruguières [Bru2000] and Müger [Müg00]. Both give a purely topological characterization of the above situation using a characterization result for neutral Tannakian categories obtained by Doplicher and Roberts [DR89 and Deligne [Del90, respectively. Both authors describe how to construct the modular braided tensor category $\mathcal{C}(W)$ from $\mathcal{C}(V)$ and the representation subcategory for the group $G$. Müger also proves the Galois correspondence, see Müg00], Sec. 4.2.

As a final example, we completely solve the extension problem for self-dual framed vertex operator algebras (FVOAs). FVOAs were introduced in [DGH98 and are VOAs which have 
a tensor product $L_{1 / 2}^{\otimes r}(0)$ of $r$ central charge $1 / 2$ rational Virasoro VOAs as subVOA with the same Virasoro element, i.e., they are the extensions of $L_{1 / 2}^{\otimes r}(0)$. Recall that $L_{1 / 2}(0)$ has three irreducible modules $L_{1 / 2}(0), L_{1 / 2}(1)$ and $L_{1 / 2}(2)$ of conformal weight $0, \frac{1}{2}$ and $\frac{1}{16}$ and $L_{1 / 2}(1)$ is a simple current. From the decomposition

$$
V=\bigoplus_{h_{1}, \ldots, h_{r} \in\{0,1,2\}} n_{h_{1}, \ldots, h_{r}} L_{1 / 2}\left(h_{1}\right) \otimes \cdots \otimes L_{1 / 2}\left(h_{r}\right)
$$

one gets the two codes

$$
\mathcal{C}=\left\{c \in\{0,1\}^{r} \mid n_{c_{1}, \ldots, c_{r}} \neq 0\right\} \text { and } \mathcal{D}=\left\{d \in\{0,1\}^{r} \mid \sum_{\substack{h_{1}, \ldots, h_{r} \\ h_{i}=2 \text { if } d_{i}=1}} n_{h_{1}, \ldots, h_{r}} \neq 0\right\}
$$

and it was shown in DGH98 that this codes are linear, $\mathcal{D} \subset \mathcal{C}^{\perp}\left(C^{\perp}\right.$ denotes the code orthogonal to $C), \mathcal{C}$ is even, and all weights of vectors in $\mathcal{D}$ are divisible by 8 .

Theorem 4.3 says that there is a unique FVOA of the form $V_{\mathcal{C}}=\bigoplus_{c \in \mathcal{C}} L_{1 / 2}\left(c_{1}\right) \otimes$ $\cdots \otimes L_{1 / 2}\left(c_{r}\right)$ for every linear even code $\mathcal{C}$, a result proven in DGH98 (Prop. 2.16) and also obtained in Miy96. As mentioned above, the associated modular category for $V_{\mathcal{C}}$ is described in Müg00. The set of irreducible modules is described in Prop. 5.2 there, a result first proven in Miy98]. In special cases, the fusion algebra and the $S$-matrix and intertwining algebra were determined in Lam2000, Lam2001.

Let now $W$ be a self-dual FVOA. From Theorem 3.3, one gets $16 \mid r$, Th. 2.19 of DGH98] gives $\mathcal{D}=\mathcal{C}^{\perp}$, and one has $\mathbf{1}^{r} \in \mathcal{D}$ for the all-one-vector.

Theorem 4.5 (Relative Mass formula for FVOAs, [Höh]) For $16 \mid r$, one has

$$
\sum_{V} \frac{1}{\left|\operatorname{Aut}_{L_{1 / 2}^{\otimes r}(0)}(V)\right|}=\frac{1}{2^{r} \cdot r !} \cdot \sum_{k} 2^{\frac{k(k-1)}{2}+1} \sigma_{k}(r),
$$

where the sum on the left hand side of the equation runs over all isomorphism classes $V$ of self-dual FVOAs of central charge $r / 2$ and $\sigma_{k}(r)$ is the number of nonequivalent codes $\mathcal{D}$ of length $r$ with $\operatorname{dim} \mathcal{D}=k, \mathbf{1}^{r} \in \mathcal{D}$ and $\operatorname{wt}(\mathcal{D}) \subset 8 \mathbf{Z}$.

Sketch of proof: Since $V_{\mathcal{C}}$ is a simple current extension, its modular category $\mathcal{T}\left(V_{\mathcal{C}}\right)$ can be expressed completely in terms of the known modular category $\mathcal{T}\left(L_{1 / 2}^{\otimes r}(0)\right)=$ $\prod_{i=1}^{r} \mathcal{T}\left(L_{1 / 2}^{\otimes r}(0)\right)$ and the code $\mathcal{C}$. For $V$ self-dual, all quantum dimensions of $\mathcal{T}\left(V_{\mathcal{C}}\right)$ are 1 , so it is an abelian modular category $\mathcal{T}(A, q)$ with an explicitly given abelian group $A$ and quadratic form $q$. One has a short exact sequence

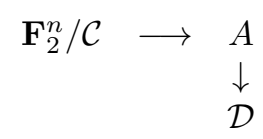

and the self-dual extensions of $V_{\mathcal{C}}$ are in one-to-one correspondence with sections $s: \mathcal{D} \longrightarrow A$ for which $s(\mathcal{D}) \subset(A, q)$ is isotropic. Counting them gives the result.

Let us check the theorem for the first case $r=16$. In GH2001, the five FVOAs of central charge 8 together with their automorphism group have been determined. For 
every $k=1,2,3,4,5$, there is an up to equivalence unique code $\mathcal{D}_{k}$ of dimension $k$ and a unique FVOA $V_{k}$ with code $\mathcal{D}\left(V_{k}\right)=\mathcal{D}_{k}$ and automorphism group $\operatorname{Aut}_{L_{1 / 2}^{\otimes 16}(0)}\left(V_{k}\right)=$ $G_{\mathcal{C}}^{k} \cdot \operatorname{Aut}\left(\mathcal{D}_{k}\right)$. By GH2001, main Theorem 2, one has $\left|G_{\mathcal{C}}^{k}\right|=2^{15-k(k+1) / 2+k}$ and for the $\operatorname{sum} \sum_{V} \frac{1}{\operatorname{Aut}_{L_{1 / 2} \otimes 16}(V) \mid}$ we obtain:

$$
\sum_{k=1}^{5} \frac{1}{\left|\operatorname{Aut}_{L_{1 / 2}^{\otimes 16}(0)}\left(V_{k}\right)\right|}=\frac{1}{2^{16} \cdot 16 !} \sum_{k=1}^{5} 2^{1+k(k+1) / 2-k} \cdot \frac{16 !}{\left|\operatorname{Aut}\left(\mathcal{D}_{k}\right)\right|},
$$

in agreement with Theorem 4.5 .

The methods of the proof can be used to give a construction of the Moonshine module $V^{\natural}$ as the self-dual FVOA with $\mathcal{C}=\mathcal{D}^{\perp}$ the lexicographic code of length 48 and minimal weight 4; see [DGH98], Sect. 5 for the precise structure of the Virasoro module decomposition. The only input from VOA-theory which one needs is the construction of the Virasoro VOA of central charge 1/2 and the structure of its intertwining algebra. All previous constructions use at some place the lattice vertex operator algebra construction; cf. [FLM88, Hua96a, Miy].

\section{References}

[AM88] Greg Anderson and Greg Moore, Rationality in Conformal Field Theory, Commun. Math. Phys. 117 (1988), 441-450.

[Ati90] Michael Atiyah, The geometry and physics of knots, Lezioni Lincee, Cambridge University Press, Cambridge, 1990.

[Ban2001] P. Bantay, The kernel of the modular representation and the Galois action in RCFT, preprint, math.QA/0102149 (2001).

[BD] A. Beilinson and V. Drinfeld, Chiral algebras, preprint.

[Boj2001] Bojko Bakalov and Alexander Kirillov, Lectures on Tensor Categories and Modular Functor, University Lecture Series 21, American Mathematical Society, Providence, RI, 2001.

[Bor86] R. E. Borcherds, Vertex algebras, Kac-Moody algebras, and the Monster, Proc. Natl. Acad. Sci. USA 83 (1986), 3068-3071.

[Bor90] R. E. Borcherds, The [fake] monster Lie algebra, Adv. math. 83 (1990), 30-47.

[Bor92] R. E. Borcherds, Monstrous moonshine and monstrous Lie superalgebras, Invent. math. 109 (1992), 405-444.

[Bru2000] A. Bruguières, Catégories prémodulaires, modularisations et invariants des variétés de dimension 3, Math. Annalen 316 (2000), 215-236.

[Cas78] J. W. S. Cassels, Rational Quadratic Forms, Academic Press, London, 1978.

[CG99] A. Coste and T. Gannon, Congruence subgroups and rational conformal field theory, preprint, math.QA/9909080 (1999).

[Del90] P. Deligne, Catégories tannakiennes, The Grothendieck Festschrift, Vol. II, Birkhäuser Boston, Boston, MA, 1990, pp. 111-195.

[DGH98] Chonying Dong, Robert Griess, and Gerald Höhn, Framed Vertex Operator Algebras, Codes and the Moonshine Module, Comm. Math. Phys. 193 (1998), 407-448, q-alg/9707008. 
[DGM90a] L. Dolan, P. Goddard, and P. Montague, Conformal Field Theory of Twisted Vertex Operators, Nuclear Physics B 338 (1990), 529-601.

[DGM90b] L. Dolan, P. Goddard, and P. Montague, Conformal Field Theory, Triality and the Monster Group, Physical Letters B 236 (1990), 165-172.

[DL93] Chongying Dong and James Lepowsky, Generalized Vertex Algebras and Relative Vertex Operators, Progress in Mathematics, Birkhäuser, Boston, 1993.

[DM96] Chongying Dong and Geoffrey Mason, Vertex operator algebras and Moonshine: a survey, Progress in algebraic combinatorics (Fukuoka, 1993), Math. Soc. Japan, Tokyo, 1996, pp. 101-136.

[DM97] Chongying Dong and Geoffrey Mason, On Quantum Galois Theory, Duke Math. J. 86 (1997), 305-321.

[DML98] Chongying Dong, Haisheng Li, and Geoffrey Mason, Twisted representations of vertex operator algebras, Math. Ann. 310 (1998), 571-600.

[DML2000] Chongying Dong, Haisheng Li, and Geoffrey Mason, Modular-invariance of trace functions in orbifold theory and generalized Moonshine, Comm. Math. Phys. 214 (2000), $1-56$.

[Don94] Chongying Dong, Representations of the moonshine module vertex operator algebra, Contemp. Math. 175 (1994), 27-36.

[DR89] Sergio Doplicher and John E. Roberts, A new duality theory for compact groups, Invent. Math. 98 (1989), 157-218.

[Dri87] V. G. Drinfel'd, Quantum groups, Proceedings of the International Congress of Mathematicians, Vol. 1, 2 (Berkeley, Calif., 1986) (Providence, RI), Amer. Math. Soc., 1987, pp. 798-820.

[DVVV89] Robbert Dijkgraaf, Cumrun Vafa, Erik Verlinde, and Herman Verlinde, The operator algebra of orbifold models, Comm. Math. Phys. 123 (1989), 485-526.

[DW90] Robbert Dijkgraaf and Edward Witten, Topological gauge theories and group cohomology, Comm. Math. Phys. 129 (1990), 393-429.

[Eho95] Wolfgang Eholzer, Fusion Algebras and Characters of Rational Conformal Field Theories, Ph.D. thesis, Universität Bonn, 1995.

[Fal94] Gerd Faltings, A proof for the Verlinde formula, J. Algebraic Geom. 3 (1994), 347-374.

[FBZ2001] Edward Frenkel and David Ben-Zvi, Vertex Algebras and Algebraic Curves, Mathemtical Surveys and Monographs, Vol. 88, Amer. Math. Soc., Providence, 2001.

[FHL93] Igor B. Frenkel, Yi-Zhi Huang, and James Lepowsky, On Axiomatic Approaches to Vertex Operator Algebras and Modules, Memoirs of the AMS, Band 104, Nr. 494, American Mathmeatical Society, Providence, 1993.

[Fin96] M. Finkelberg, An equivalence of fusion categories, Geom. Funct. Anal. 6 (1996), 249267.

[FK93] Jürg Fröhlich and Thomas Kerler, Quantum groups, quantum categories and quantum field theory, Lecture Notes in Mathematics 1542, Springer-Verlag, Berlin, 1993.

[FLM88] Igor Frenkel, James Lepowsky, and Arne Meuerman, Vertex Operator Algebras and the Monster, Academic Press, San Diego, 1988.

[FQ93] Daniel S. Freed and Frank Quinn, Chern-Simons theory with finite gauge group, Comm. Math. Phys. 156 (1993), 435-472.

[FSS96] J. Fuchs, A. N. Schellekens, and C. Schweigert, A matrix S for all simple current extensions, Nuclear Phys. B 473 (1996), 323-366. 
[Fuc94] Jürgen Fuchs, Fusion rules in conformal field theory, Fortschr. Phys. 42 (1994), 1-48.

[FZ92] Igor B. Frenkel and Yongchang Zhu, Vertex Operator Algebras associated to Representations of Affine and Virasoro Algebras, Duke Mathematical Journal 66 (1992), $123-168$.

[Gau1801] C. F. Gauss, Disquisitiones Arithmeticae, Leipzig, 1801.

[GH2001] Robert Griess and Gerald Höhn, Virasoro Frames and their Stabilizers for the $E_{8}$ Lattice type Vertex Operator Algebra, to appear in Journal für die reine und angewandte Mathematik, math.QA/0101054 (2001).

[Gri98] Robert L. Griess, Jr., A vertex operator algebra related to $E_{8}$ with automorphism group $\mathrm{O}^{+}(10,2)$, The Monster and Lie algebras (Columbus, OH, 1996), de Gruyter, Berlin, 1998, pp. $43-58$.

[HL94] Yi-Zhi Huang and James Lepowsky, Tensor Products of Modules for a Vertex Operator Algebra and Vertex Tensor Categories, Progress in Math., Vol. 123, Birkhäuser, Boston, 1994, pp. 349-383.

[HL99] Yi-Zhi Huang and James Lepowsky, Intertwining operator algebras and vertex tensor categories for affine Lie algebras, Duke Math. J. 99 (1999), 113-134.

[HM99] Yi-Zhi Huang and Antun Milas, Intertwining operator superalgebras and vertex tensor categories for superconformal algebras, I, preprint, math.QA/9909039 (1999).

[HM2002] Yi-Zhi Huang and Antun Milas, Intertwining operator superalgebras and vertex tensor categories for superconformal algebras, II, Trans. Amer. Math. Soc. 354 (2002), 363385.

[HMT99] Akihide Hanaki, Masahiko Miyamoto, and Daisuke Tambara, Quantum Galois theory for finite groups, Duke Math. J. 97 (1999), 541-544.

[Höh] Gerald Höhn, A mass formula for framed vertex operator algebras, in preparation.

[Höh95] Gerald Höhn, Selbstduale Vertexoperatorsuperalgebren und das Babymonster, Ph.D. thesis, Universität Bonn, 1995, see: Bonner Mathematische Schriften 286.

[Höh97] Gerald Höhn, Self-dual Vertex Operator Superalgebras with Shadows of large minimal weight, Internat. Math. Res. Notices 13 (1997), 613-621, q-alg/9608023.

[HS2001] Gerald Höhn and Nils R. Scheithauer, A natural construction of Borcherds' fake Baby Monster Lie Algebra, preprint 2001.

[Hua96a] Yi-Zhi Huang, A nonmeromorphic extension of the Moonshine module vertex operator algebra, Moonshine, the Monster, and related topics (South Hadley, MA, 1994), Amer. Math. Soc., Providence, RI, 1996, hep-th/9406190, pp. 123-148.

[Hua96b] Yi-Zhi Huang, Virasoro vertex operator algebras, the (nonmeromorphic) operator product expansion and the tensor product theory, J. Algebra 182 (1996), 201-234.

[Hua97] Yi-Zhi Huang, Two-Dimensional Conformal Geometry and Vertex Operator Algebras, Progress in Mathematics, 148, Birkhäuser, Boston, Basel, Berlin, 1997.

[Kac97] Victor Kac, Vertex algebras for beginners, University Lecture Series, vol. 10, American Mathematical Society, Providence, RI, 1997.

[Kir78] Robion Kirby, A calculus for framed links in $S^{3}$, Invent. Math. 45 (1978), 35-56.

[KL91] David Kazhdan and George Lusztig, Affine Lie algebras and quantum groups, Internat. Math. Res. Notices (1991), no. 2, 21-29.

[Kne57] Martin Kneser, Klassenzahlen definiter quadratische Formen, Archiv der Mathematik 8 (1957), 241-250. 
[KO2001] Alexander Kirillov and Viktor Ostrik, On q-analog of McKay correspondence and ADE classification of $\widehat{s l}_{2}$ conformal field theories, preprint, math.QA/0101219 (2001).

[Kud84] Stephen S. Kudla, Seesaw dual reductive pairs, Automorphic forms of several variables (Katata, 1983), Birkhäuser Boston, Boston, MA, 1984, pp. 244-268.

[Lam2000] Ching Hung Lam, Some twisted modules for framed vertex operator algebras, J. Algebra 231 (2000), 331-341.

[Lam2001] Lam, Ching Hung, Fusion rules for the Hamming code vertex operator algebra, Comm. Algebra 29 (2001), 2125-2145.

[Mac52] Saunders MacLane, Cohomology theory of Abelian groups, Proceedings of the International Congress of Mathematicians, Cambridge, Mass., 1950, vol. 2 (Providence, R. I.), Amer. Math. Soc., 1952, pp. 8-14.

[Min1884] H. Minkowski, Grundlagen für eine Theorie der quadratischen Formen mit ganzzahligen Koeffizienten, Mém. près. par divers savants á l'Acadèmie des Sci. Inst. nat. de France 29 (1884).

[Miy] Masahiko Miyamoto, A new construction of the Moonshine vertex operator algebra over the real number field, preprint.

[Miy96] Masahiko Miyamoto, Binary codes and vertex operator (super)algebras, J. Algebra 181 (1996), 207-222.

[Miy98] Masahiko Miyamoto, Representation theory of code vertex operator algebra, J. Algebra 201 (1998), 115-150.

[MN2001] Geoffrey Mason and Siu-Hung Ng, Group cohomology and gauge equivalence of some twisted quantum doubles, Trans. Amer. Math. Soc. 353 (2001), 3465-3509.

[MS89] Gregory Moore and Nathan Seiberg, Classical and Quantum Conformal Field Theory, Commun. Math. Phys. 123 (1989), 177-254.

[Müg00] Michael Müger, Galois Theory for Braided Tensor Categories and the Modular Closure, Adv. Math. 150 (2000), 151-201, math.CT/9812040v3.

[Nik80] V. V. Nikulin, Integral symmetric bilinear forms and some of their applications, Math. USSR Izvestija 14 (1980), 103-167.

[Ray01] Urmie Ray, A short note on automorphisms of generalized Kac-Moody superalgebras, preprint (2001).

[Ros01] Markus Rosellen, Abelian intertwining algebras, preprint (2001).

[RT91] N. Reshetikhin and V. G. Turaev, Invariants of 3-manifolds via link polynomials and quantum groups, Invent. Math. 103 (1991), 547-597.

[Sch93] A. N. Schellekens, Meromorphic $c=24$ Conformal Field Theories, Comm. Math. Phys. 153 (1993), 159-185.

[Seg89] Graeme Segal, The Definition of Conformal Field Theory, preprint (1989).

[Sie35] C. L. Siegel, Über die analytische Theorie der quadratischen Formen, Annals of Mathematics 36 (1935), 527-606.

[Smi1867] H. J. S. Smith, On the orders and genera of quadratic forms containing more than three indeterminates, Proc. R. Soc. London 16 (1867), 197-208.

[Tur94] V. G. Turaev, Quantum Invariants of Knots and 3-Manifolds, de Gruyter Studies in Mathematics, Walter de Gruyter, Berlin, New York, 1994.

[TUY89] Akihiro Tsuchiya, Kenji Ueno, and Yasuhiko Yamada, Conformal field theory on universal family of stable curves with gauge symmetries, Integrable systems in quantum field theory and statistical mechanics, Adv. Stud. Pure Math., 19, Academic Press, Boston, MA, 1989, pp. 459-566. 
[Vaf88] Cumrun Vafa, Toward classification of conformal theories, Phys. Lett. B 206 (1988), 421-426.

[Wei65] Andre Weil, Sur la formule de Siegel dans la théorie des groupes classiques, Acta Math. 113 (1965), 1-87.

[Wit89] Edward Witten, Quantum field theory and the Jones Polynomial, Commun. Math. Phys. 121 (1989), 351-399.

[Zhu90] Yongchang Zhu, Vertex Operator Algebras, Elliptic Functions, and Modular Forms, Ph.D. thesis, Yale University, 1990, appeared as: Modular invariance of characters of vertex operator algebras, J. Amer. Math. Soc 9 (1996).

[Zhu94] Yongchang Zhu, Global Vertex Operators on Riemann Surfaces, Comm. Math. Physics 165 (1994), 485-531. 